\title{
Pólya-type polynomial inequalities in Orlicz spaces and best local approximation
}

\author{
Héctor H Cuenya ${ }^{1}$, Fabián E Levis ${ }^{1}$ and Claudia V Ridolfi ${ }^{*}$
}

* Correspondence: ridolfi@unsl.edu. ar

${ }^{2}$ Department of Mathematics, UNSL, 5700, San Luis, Argentina Full list of author information is available at the end of the article

\begin{abstract}
We obtain an extension of Pólya-type inequalities for univariate real polynomials in Orlicz spaces. We also give an application to a best local approximation problem. MSC 2010: 41A10; $41 \mathrm{~A} 17$.

Keywords: algebraic polynomials, p?ó?lya-type inequalities, best local approximation, balanced integers
\end{abstract}

\section{Introduction}

Let $X$ be a bounded open subset of $\mathbb{R}$. Consider the measure space $(X, \mathcal{B}, \mu)$, where $\mu$ is the Lebesgue measure, and denote $\mathcal{M}=\mathcal{M}(X)$ the system of all equivalence classes of Lebesgue measurable real valued functions on $X$. Let $\Phi$ be the set of convex functions $\varphi: \mathbb{R}_{+} \rightarrow \mathbb{R}_{+}$, with $\varphi(x)>0$ for $x>0$, and $\varphi(0)=0$.

Given $\varphi \in \Phi$, we define

$$
L^{\phi}=L^{\phi}(X):=\left\{f \in \mathcal{M}: \int_{X} \phi(\alpha|f(x)|) d x<\infty, \text { for some } \alpha>0\right\} .
$$

The space $L^{\varphi}$ is called the Orlicz space determined by $\varphi$. This space is endowed with the Luxemburg norm,

$$
\|f\|_{\phi, X}=\inf \left\{\lambda>0: \int_{X} \phi\left(\frac{|f(x)|}{\lambda}\right) \frac{d x}{\mu(X)} \leq 1\right\} .
$$

The space $L^{\varphi}$ with this norm is a Banach space (see [1]). If $E \in \mathcal{B}$ and $\mu(E)>0$, then $\|\cdot\|_{\varphi, E}$ is a seminorm on $L^{\varphi}(X)$. In the particular case, $\varphi(t)=t^{p}$, we will use the notation $\|\cdot\|_{p, E}$ instead of $\|\cdot\|_{\varphi, E}$.

Let $\Pi^{N} \subset \mathcal{M}, N \in \mathbb{N}$, be the class of all algebraic polynomials of degree at most $N$, with real coefficients.

Given $E \in \mathcal{B}$, we recall that a polynomial $g_{E} \in \Pi^{N}$ is a best approximation of $f \in L^{\varphi}$ $(X)$ from $\Pi^{N}$ respect to $\|\cdot\|_{\varphi, E}$, if

$$
\left\|f-g_{E}\right\|_{\phi, E}=\inf \left\{\|f-P\|_{\phi, E}: \quad P \in \prod^{N}\right\} .
$$

(C) 2012 Cuenya et al.; licensee Springer. This is an open access article distributed under the terms of the Creative Commons Attribution License (http://creativecommons.org/licenses/by/2.0), which permits unrestricted use, distribution, and reproduction in any medium, provided the original work is properly cited. 
Let $x_{k}, 1 \leq k \leq n$, be $n$ points in $X$. We consider a net of measurable sets $\{E\} \subset \mathcal{B}$ such that $E=\bigcup_{k=1}^{n} E_{k}$, with $\mu\left(E_{k}\right)>0$ and

$$
\sup _{1 \leq k \leq n} \sup _{\gamma \in E_{k}}\left|x_{k}-\gamma\right| \rightarrow 0, \quad \text { as } \mu(E) \rightarrow 0 .
$$

Given $f \in L^{\varphi}(X)$ and $\Pi^{N}$, we consider a net of best approximation functions $\left\{g_{E}\right\}$. If it has a limit in $\Pi^{N}$ as $\mu(E) \rightarrow 0$, this limit is called the best local approximation of $f$ from $\Pi^{N}$ on $\left\{x_{1}, \ldots, x_{n}\right\}$. If the points in our approximation problem have not the same importance the neighborhoods $E_{k}$ can be adjusted to reflect it. In [2], Chui et al. introduced the balanced neighborhood concept and they studied existence and characterization of best local approximation in $L^{p}$-spaces for several points with different size neighborhoods. In $[3,4]$, the last problem was considered for $\varphi$-approximation and $\|\cdot\|_{\varphi^{-}}$ approximation, respectively, in Orlicz spaces. Other results in these spaces about best local approximation with non balanced neighborhoods were considered in [5].

Polynomial inequalities on measurable sets have been studied extensively in the literature (see [6-8]). In [9], the authors proved the following extension of the Pólya inequality in $L^{p}$-spaces, $0<p \leq \infty$.

Theorem 1.1. Let $0<p \leq \infty$ and $n, N \in \mathbb{N}$. Let $i_{k}, 1 \leq k \leq n$, be $n$ positive integers such that $\sum_{k=1}^{n} i_{k}=N+1$. Let $B_{k}, 1 \leq k \leq n$, be disjoint pairwise compact intervals in $\mathbb{R}$ with $0<\mu\left(B_{k}\right) \leq 1$. Then there exists a constant $K$ depending on $p, i_{k}$ and $B_{k}$, for $1 \leq$ $k \leq n$, such that

$$
\left|c_{j}\right| \leq \frac{K}{\min _{1 \leq k \leq n} \mu\left(E \cap B_{k}\right)^{i_{k}-1+1 / p}}\|P\|_{p, E,} \quad 0 \leq j \leq N,
$$

for all $P(x)=\sum_{j=0}^{N} c_{j} x^{j}, E \subset \bigcup_{k=1}^{n} B_{k}, \mu\left(E \cap B_{k}\right)>0, \quad 1 \leq k \leq n$.

They gave an application of this theorem to the existence of the best multipoint local approximation in $L^{p}$ spaces, with balanced neighborhoods.

In this article, we generalize Theorem 1.1 and the balanced neighborhood concept to $L^{\varphi}$. As a consequence of this extension we prove the existence of the best local approximation of a function from $\Pi^{N}$ on $\left\{x_{1}, \ldots, x_{n}\right\}$, with balanced neighborhoods, following the pattern used in [9]. Moreover, we prove that the best local approximation polynomial is the Hermite interpolating polynomial.

We say that a function $\varphi \in \Phi$ satisfies the $\Delta_{2}$-condition if there exists a constant $k>$ 0 such that $\varphi(2 x) \leq k \varphi(x)$, for $x \geq 0$, and we say that $\varphi$ satisfies the $\Delta^{\prime}$-condition if there exists a constant $c>0$ such that $\varphi(x y) \leq c \varphi(x) \varphi(y)$ for $x, y \geq 0$. We point out that the $\Delta^{\prime}$-condition implies the $\Delta_{2}$-condition. A detailed treatment about these subjects may be found in [1].

If $\varphi$ satisfies the $\Delta^{\prime}$-condition, it is easy to see that there exists a constant $K>0$ such that

$$
\phi^{-1}(x) \phi^{-1}(y) \leq K \phi^{-1}(x y), \quad \text { for all } x, y \geq 0 .
$$

We assume in this article that $\varphi \in \Phi$ and it satisfies the $\Delta^{\prime}$-condition.

\section{Preliminary results}

Let $\mathcal{X}_{A}$ denotes the characteristic function on the measurable set $A \subset X$. 
Proposition 2.1. The family of all seminorms $\|\cdot\|_{\varphi . E}$ with $\mu(E)>0$, has the following properties:

(a) $\left\|\mathcal{X}_{E}\right\|_{\phi, E}=\frac{1}{\phi^{-1}(1)}$.

(b) if $f, g \in L^{\varphi}(X)$ satisfy $|f| \leq|g|$ on $E$, then $\|f\|_{\varphi, E} \leq\|g\|_{\varphi, E}$. The inequality is strict if $|f|<|g|$ on some subset of $E$ with positive measure.

(c) There exists a constant $M>0$ such that

$$
\|f\|_{\phi, G} \leq \frac{M}{\phi^{-1}\left(\frac{\mu(G)}{\mu(D)}\right)}\|f\|_{\phi, D,} \quad f \in L^{\phi}(X),
$$

for all pair of measurable sets $G, D$, with $G \subset D$ and $\mu(G)>0$.

Proof (a) For $\lambda:=1 / \varphi^{-1}(1)$ we have

$$
\int_{E} \phi\left(\frac{\left|\mathcal{X}_{E}\right|}{\lambda}\right) \frac{d x}{\mu(E)}=\int_{E} \frac{d x}{\mu(E)}=1 .
$$

Now, the $\Delta_{2}$ - condition implies $\left\|\mathcal{X}_{E}\right\|_{\phi, E}=1 / \phi^{-1}(1)$.

(b) If $|f| \leq|g|$ on $E$, then

$$
\int_{E} \phi\left(\frac{|f|}{\lambda}\right) \frac{d x}{\mu(E)} \leq \int_{E} \phi\left(\frac{|g|}{\lambda}\right) \frac{d x}{\mu(E)}, \quad \lambda>0,
$$

and so $\|f\|_{\varphi, E} \leq\|g\|_{\varphi, E}$. In addition, if $|f|<|g|$ on some subset of $E$ with positive measure, the above inequality is strict. So, the $\Delta_{2}$-condition implies the assertion.

(c) Given $G \subset D, \mu(G)>0$, and $f \in L^{\varphi}(X)$, for each $\lambda>0$, we denote

$$
\mathfrak{A}(\lambda):=\int_{G} \phi\left(\frac{|f|}{\lambda}\right) \frac{d x}{\mu(G)} \text { and } \mathfrak{B}(\lambda):=\int_{D} \phi\left(\frac{|f|}{\lambda}\right) \frac{d x}{\mu(D)} .
$$

We consider $\lambda>0$ such that $\mathfrak{B}(\lambda) \leq 1$. By the $\Delta^{\prime}$-condition we obtain

$$
\mathfrak{A}\left(\frac{\lambda}{\phi^{-1}\left(\frac{\mu(G)}{c \mu(D)}\right)}\right) \leq \int_{D} c \frac{\mu(G)}{c \mu(D)} \phi\left(\frac{|f|}{\lambda}\right) \frac{d x}{\mu(G)}=\mathfrak{B}(\lambda) \leq 1 .
$$

Then $\|f\|_{\phi, G} \leq \frac{\lambda}{\phi^{-1}\left(\frac{\mu(G)}{c \mu(D) \mid}\right)}$, for all $\lambda>0$ with $\mathfrak{B}(\lambda) \leq 1$. So, the definition of $\|f\|_{\varphi, D}$ and (1) imply $\|f\|_{\phi, G} \leq \frac{M}{\phi^{-1}\left(\frac{\mu(G)}{\mu(D)}\right)}\|f\|_{\phi, D}$ with $M=\frac{K}{\phi^{-1}\left(c^{-1}\right)}$.

Lemma 2.2. There exists a constant $M>0$ such that

$$
\left|P^{(j)}(a)\right| \leq \frac{M}{\varepsilon^{j}}\|P\|_{\phi,[a-\varepsilon, a+\varepsilon]}
$$


for all $P \in \Pi^{N},[a-\epsilon, a+\epsilon] \subset X$, and $0 \leq j \leq N$.

Proof. Given $P \in \Pi^{N}$ and $[a-\epsilon, a+\epsilon] \subset X$, we divide that interval in $2(N+1)$ close subintervals with the same size. Let $J_{\epsilon}$ be one of them. From Proposition 2.1 (c), we get $\|P\|_{\phi, J_{\varepsilon}} \leq M\|P\|_{\phi,[a-\varepsilon, a+\varepsilon]}$, where $M$ is independent on $P$, $a$, and $\epsilon$. In addition, there exists $y_{\epsilon} \in J_{\epsilon}$ such that $\left|P\left(y_{\varepsilon}\right)\right| \leq \phi^{-1}(1)\|P\|_{\phi_{I} J_{\varepsilon}}$. In fact, if $\phi^{-1}(1)\|P\|_{\phi_{J_{\varepsilon}}}<|P(y)|$, for all $y \in J_{\epsilon}$, then Proposition 2.1 (a) and (b) yield $\|P\|_{\phi_{J_{\varepsilon}}}>\|P\|_{\phi_{I} J_{\varepsilon}}$. A contradiction.

From the family of intervals $J_{\epsilon}$, we choose pairwise disjoint $(N+1)$ intervals, and we denote them with $J_{i, \epsilon}, 1 \leq i \leq N+1$. Let $y_{i, \epsilon} \in J_{i, \epsilon}$ be such that

$$
\left|P\left(\gamma_{i, \varepsilon}\right)\right| \leq M \phi^{-1}(1)\|P\|_{\phi,[a-\varepsilon, a+\varepsilon],} \quad 1 \leq i \leq N+1 .
$$

If $t_{i, \varepsilon}:=\frac{y_{i, \varepsilon}-a}{\varepsilon} \in[-1,1]$, we have

$$
P\left(\gamma_{i, \varepsilon}\right)=\sum_{j=0}^{N} \frac{P^{(j)}(a)}{j !}\left(\gamma_{i, \varepsilon}-a\right)^{j}=\sum_{j=0}^{N} \frac{P^{(j)}(a)}{j !} \varepsilon^{j} t_{i, \varepsilon^{\prime}}^{j} \quad 1 \leq i \leq N+1 .
$$

The matrix of the linear system (4), $\left(t_{i, \varepsilon}^{j}\right)$, is a Vandermonde matrix whose determinant has a positive lower bound, because $t_{i, \epsilon}-t_{i^{\prime}, \epsilon} \geq 1 / N+1$ for $i>i^{\prime}$. Using Cramer's rule and (3), there is a constant which we again denote by $M$ such that

$$
\left|P^{(j)}(a) \varepsilon^{j}\right| \leq M\|P\|_{\phi,[a, \varepsilon, a+\varepsilon]} \quad 0 \leq j \leq N .
$$

The proof of the following lemma is analogous to the one of Lemma 2.3 in [9], however we give it for sake of completeness.

Lemma 2.3. Let $C \subset X$ be an interval, $E \subset C, \mu(E)>0$. For all $P \in \Pi^{N}$, there exists an interval $F:=F(E, P) \subset C$ such that
a) $\mu(F) \geq \frac{\mu(E)}{2 N}$,
b) $\|P\|_{\varphi, F} \leq 2 N\|P\|_{\varphi, E}$.

Proof. Let $P \in \Pi^{N}, S=2 N$, and let $D_{a}:=\{x \in C:|P(x)|<a\}$. It easy to see that the function $G(a):=\mu\left(D_{a}\right)$ is continuous, $G(0)=0$ and $\lim _{a \rightarrow \infty} G(a)=\mu(C)$. Therefore, there exists a constant $a_{*} \in \mathbb{R}^{+}$such that $\mu\left(D_{a_{*}}\right)=\mu(E) / 2$. Since $\left\{x \in C:|P(x)|=a_{*}\right\}$ has at most $2 N$ elements, there exists $k, 1 \leq k \leq N$, and pairwise disjoint intervals $E_{j}, 1 \leq j \leq$ $k$, such that $D_{a_{*}}=\bigcup_{j=1}^{k} E_{j}$.

We denote $\bar{A}=C \backslash A$, for any set $A$. Then

$$
\mu\left(E \cap \bar{D}_{a_{*}}\right)=\mu(E)-\mu\left(E \cap D_{a_{*}}\right) \geq \mu(E)-\mu\left(D_{a_{*}}\right)=\frac{\mu(E)}{2} .
$$

There exists $j, 1 \leq j \leq k$, such that $\mu\left(E_{j}\right) \geq \mu(E) / S$. In fact, if $\mu\left(E_{j}\right)<\mu(E) / S$ for all $j, 1 \leq$ $j \leq k$, we obtain $\mu\left(D_{a_{*}}\right)<k / S \mu(E) \leq \mu(E) / 2$, which is a contradiction. So, we have proved a) with $F:=E_{j}$. 
Using (5), we obtain

$$
\mu\left(E \cap \bar{D}_{a_{*}}\right) \geq \frac{\mu(E)}{2}=\mu\left(D_{a_{*}}\right) \geq \mu(F) \mu(F \cap \bar{E})
$$

Therefore

$$
\begin{aligned}
\int_{F} \phi\left(\frac{|P|}{\lambda}\right) \frac{d x}{\mu(F)} & \leq \int_{F \cap E} \phi\left(\frac{|P|}{\lambda}\right) \frac{d x}{\mu(F)}+\phi\left(\frac{a_{*}}{\lambda}\right) \frac{\mu\left(E \cap \bar{D}_{a_{*}}\right)}{\mu(F)} \\
& \leq \int_{E \cap D_{a_{*}}} \phi\left(\frac{|P|}{\lambda}\right) \frac{d x}{\mu(F)}+\int_{E \cap \bar{D}_{a_{*}}} \phi\left(\frac{|P|}{\lambda}\right) \frac{d x}{\mu(F)} \\
& =\int_{E} \phi\left(\frac{|P|}{\lambda}\right) \frac{d x}{\mu(F)} .
\end{aligned}
$$

So, (a) implies

$$
\mathcal{A}_{F}(\lambda):=\int_{F} \phi\left(\frac{|P|}{\lambda}\right) \frac{d x}{\mu(F)} \leq S \int_{E} \phi\left(\frac{|P|}{\lambda}\right) \frac{d x}{\mu(E)}=: S \mathcal{A}_{E}(\lambda) .
$$

Let $\lambda$ be such that $\mathcal{A}_{E}(\lambda)=1$. The convexity of $\varphi$ implies $\mathcal{A}_{F}(S \lambda) \leq 1$. So, $\|P\|_{\varphi, F} \leq$ $S\|P\|_{\varphi, E}$.

\section{Pólya inequality}

Now, we present the main result concerning to Pólya inequality in $L^{\varphi}$.

Theorem 3.1. Let $\varphi \in \Phi$, and $n, N \in \mathbb{N}$. Let $i_{k}, 1 \leq k \leq n$, be $n$ positive integers such that $\sum_{k=1}^{n} i_{k}=N+1$. Let $B_{k}, 1 \leq k \leq n$, be disjoint pairwise compact intervals in $\mathbb{R}$, with $0<\mu\left(B_{k}\right) \leq 1$. Then there exists a positive constant $M$ depending on $\varphi, i_{k}$, and $B_{k}, 1$ $\leq k \leq n$, such that

$$
\left|c_{j}\right| \leq \frac{M}{\min _{1 \leq k \leq n}\left\{\mu\left(E \cap B_{k}\right)^{i_{k}-1} \phi^{-1}\left(\frac{\mu\left(E \cap B_{k}\right)}{\mu(E)}\right)\right\}}\|P\|_{\phi, E,} \quad 0 \leq j \leq N,
$$

for all $P(x)=\sum_{j=0}^{N} c_{j} x^{j}, E \subset \bigcup_{k=1}^{n} B_{k}$ with $\mu\left(E \cap B_{k}\right)>0,1 \leq k \leq n$.

Proof. In the following proof, the constant $M$ can be different in each occurrence. Let $P(x)=\sum_{j=0}^{N} c_{j} x^{j} \in \Pi^{N}$, and let $E \subset \bigcup_{k=1}^{n} B_{k}$ be a measurable set with $\mu\left(E \cap B_{k}\right)>0,1$ $\leq k \leq n$. By Lemma 2.3 for $C=B_{k}$, there exist $n$ intervals $F_{k}=\left[a_{k}-r_{k}, a_{k}+r_{k}\right] \subset B_{k}, 1$ $\leq k \leq n$, such that $\mu\left(F_{k}\right) \geq \mu\left(E \cap B_{k}\right) / 2 N$ and $\|P\|_{\phi, F_{k}} \leq 2 N\|P\|_{\phi, E \cap B_{k}}$. From Lemma 2.2, there exists a positive constant $M$ depending on $p, i_{k}$, and $B_{k}, 1 \leq k \leq n$, such that for all $j, 0 \leq j \leq i_{k}-1,1 \leq k \leq n$, it verifies

$$
\left|P^{(j)}\left(a_{k}\right)\right| \leq \frac{M}{\mu\left(F_{k}\right)^{j}}\|P\|_{\phi, F_{k}} \leq \frac{M}{\mu\left(F_{k}\right)^{i_{k}-1}}\|P\|_{\phi, F_{k}} \leq \frac{M}{\mu\left(E \cap B_{k}\right)^{i_{k}-1}}\|P\|_{\phi, E \cap B_{k}} .
$$

From (7) and (2), there is a constant $M$ such that

$$
\left|P^{(j)}\left(a_{k}\right)\right| \leq \frac{M}{\mu\left(E \cap B_{k}\right)^{i_{k}-1} \phi^{-1}\left(\frac{\mu\left(E \cap B_{k}\right)}{\mu(E)}\right)}\|P\|_{\phi, E}
$$


for $0 \leq j \leq i_{k}-1,1 \leq k \leq n$. So

$$
\left|P^{(j)}\left(a_{k}\right)\right| \leq \frac{M}{\min _{1 \leq s \leq n}\left\{\mu\left(E \cap B_{s}\right)^{i_{s}-1} \phi^{-1}\left(\frac{\mu\left(E \cap B_{s}\right)}{\mu(E)}\right)\right\}}\|P\|_{\phi, E^{\prime}}
$$

for $0 \leq j \leq i_{k}-1,1 \leq k \leq n$. From the equivalence of the norms $\|\cdot\|_{1}$ and $\|\cdot\|_{2}$ on $\Pi^{N}$,

$$
\|P\|_{1}=\max _{1 \leq k \leq n} \sup _{a_{k} \in B_{k}} \max _{0 \leq j \leq i_{k}-1}\left|P^{(j)}\left(a_{k}\right)\right| \text { and }\|P\|_{2}=\max _{0 \leq j \leq N}\left|c_{j}\right|,
$$

we obtain (6).

\section{Best local approximation}

In this section, we introduce a concept of balanced neighborhood in $L^{\varphi}$ and we prove the existence of the best local approximation using the neighborhoods $E_{k}, 1 \leq k \leq n$, mentioned in the Section 1.

It is easy to see that $E_{k}=x_{k}+\mu\left(E_{k}\right) A_{k}$, where $A_{k}$ is a measurable set with measure 1 . Henceforward, we assume the sets $A_{k}$ are uniformly bounded.

For each $\alpha \in \mathbb{R}$ and $k, 1 \leq k \leq n$, we denote

$$
\mathcal{A}_{k}(\alpha):=\frac{\mu\left(E_{k}\right)^{\alpha}}{\phi^{-1}\left(\frac{\mu(E)}{\mu\left(E_{k}\right)}\right)} .
$$

We assume the following condition, which allows us that $\mathcal{A}_{k}(\alpha)$ can be compared with each other as functions of $\alpha$ when $\mu(E) \rightarrow 0$.

For any nonnegative integers $\alpha$ and $\beta$, and any pair $j, k, 1 \leq j, k \leq n$,

$$
\text { either } \mathcal{A}_{k}(\alpha)=O\left(\mathcal{A}_{j}(\beta)\right) \text { or } \mathcal{A}_{j}(\beta)=o\left(\mathcal{A}_{k}(\alpha)\right), \quad \text { as } \mu(E) \rightarrow 0 .
$$

Let $<i_{k}>$ be an ordered $n$-tuple of nonnegative integers. We say that $\mathcal{A}_{j}\left(i_{j}\right)$ is a $\max$ imal element of $\left\langle\mathcal{A}_{k}\left(i_{k}\right)>\right.$ if $\mathcal{A}_{k}\left(i_{k}\right)=O\left(\mathcal{A}_{j}\left(i_{j}\right)\right)$ for all $1 \leq k \leq n$. We denote it by

$$
\mathcal{A}_{j}\left(i_{j}\right)=\max \left\{\mathcal{A}_{k}\left(i_{k}\right)\right\} \text {. }
$$

Observe that $\sum_{k=1}^{n} \mathcal{A}_{k}\left(i_{k}\right)=O\left(\max \left\{\mathcal{A}_{k}\left(i_{k}\right)\right\}\right)$.

Definition 4.1. An n-tuple $<i_{k}>$ of nonnegative integers is balanced if

$$
\sum_{k=1}^{n} \mathcal{A}_{k}\left(i_{k}\right)=o\left(\min _{1 \leq k \leq n}\left\{\mu\left(E_{k}\right)^{i_{k}-1} \phi^{-1}\left(\frac{\mu\left(E_{k}\right)}{\mu(E)}\right)\right\}\right) .
$$

In this case, we say that $\sum_{k=1}^{n} i_{k}$ is a balanced integer, and $<E_{k}>$ are balanced neighborhoods.

Lemma 4.2. To each balanced integer there corresponds exactly one balanced n-tuple.

Proof. Let $\left\langle i_{k}\right\rangle$ be a balanced $n$-tuple. If $\left\langle i_{k}^{\prime}\right\rangle$ is distinct from $\left\langle i_{k}\right\rangle$ and $\sum_{k=1}^{n} i_{k}=\sum_{k=1}^{n} i_{k}^{\prime}$, there exist indices $j$ and $s$ such that $i_{j} \geq i_{j}^{\prime}+1$ and $i_{s}^{\prime} \geq i_{s}+1$. From definition of balanced neighborhood, we have

$$
\mathcal{A}:=\sum_{k=1}^{n} \mathcal{A}_{k}\left(i_{k}\right)=o\left(\mu\left(E_{j}\right)^{i_{j}-1} \phi^{-1}\left(\frac{\mu\left(E_{j}\right)}{\mu(E)}\right)\right) .
$$


In addition, by (1) we get $\mu\left(E_{j}\right)^{i_{j}-1} \phi^{-1}\left(\frac{\mu\left(E_{j}\right)}{\mu(E)}\right) \leq \mu\left(E_{j}\right)^{i_{j} \phi} \phi^{-1}\left(\frac{\mu\left(E_{j}\right)}{\mu(E)}\right) \leq K \phi^{-1}(1) \mathcal{A}_{j}\left(i_{j}^{\prime}\right)$.

So, $\mathcal{A}=o\left(\sum_{k=1}^{n} \mathcal{A}_{k}\left(i^{\prime}{ }_{k}\right)\right)$. Again, by (1) we get

$$
\frac{\sum_{k=1}^{n} \mathcal{A}_{k}\left(i_{k}{ }_{k}\right)}{\mu\left(E_{s}\right)^{i_{s}^{\prime}-1} \phi^{-1}\left(\frac{\mu\left(E_{s}\right)}{\mu(E)}\right)} \geq \frac{\sum_{k=1}^{n} \mathcal{A}_{k}\left(i^{\prime}{ }_{k}\right)}{\mu\left(E_{s}\right)^{i_{s}} \phi^{-1}\left(\frac{\mu\left(E_{s}\right)}{\mu(E)}\right)} \geq \frac{\sum_{k=1}^{n} \mathcal{A}_{k}\left(i^{\prime}{ }_{k}\right)}{K \phi^{-1}(1) \mathcal{A}_{s}\left(i_{s}\right)} \rightarrow \infty .
$$

Then $<i_{k}^{\prime}>$ cannot be balanced.

The following lemma allows us to state an algorithm to compute all the balanced integers greater than a given balanced integer.

Lemma 4.3. Let $<i_{k}>$ and $<i_{k}^{\prime}>$ be two balanced $n$-tuples with $\sum_{k=1}^{n} i_{k}<\sum_{k=1}^{n} i_{k}^{\prime}$. Let $A=A\left(<i_{k}>\right):=\left\{j: \mathcal{A}_{j}\left(i_{j}\right)=\max \left\{\mathcal{A}_{k}\left(i_{k}\right)\right\}\right\}$ and $B=B\left(<i_{k}>\right):=\{1,2, \ldots, n\} \backslash A$. Then

(a) for $j \in A i_{j}^{\prime} \geq i_{j}+1$.

(b) for $j \in A \quad i_{j}^{\prime} \geq i_{j}$.

Proof. (a) Suppose $i_{j}^{\prime} \leq i_{j}$ for some $j \in A$. For any $l \in B$, from (8) we get $\mathcal{A}_{l}\left(i_{l}\right)=o\left(\mathcal{A}_{j}\left(i_{j}\right)\right)$. Assume now $i_{l}^{\prime} \geq i_{l}+1$ for some $l \in B$. By (1), there exists a constant $M>0$ such that

$$
\frac{\mathcal{A}_{j}\left(i_{j}^{\prime}\right)}{\mu\left(E_{l}\right)^{i^{\prime}{ }^{\prime}-1} \phi^{-1}\left(\frac{\mu\left(E_{l}\right)}{\mu(E)}\right)} \geq \frac{\mathcal{A}_{j}\left(i_{j}\right)}{\mu\left(E_{l}\right)^{i_{l}} \phi^{-1}\left(\frac{\mu\left(E_{l}\right)}{\mu(E)}\right)} \geq \frac{\mathcal{A}_{j}\left(i_{j}\right)}{M \mathcal{A}_{l}\left(i_{l}\right)} \rightarrow \infty,
$$

as $\mu(E) \rightarrow 0$. Thus $<i_{k}^{\prime}>$ cannot be balanced, a contradiction. Therefore, either $B=\emptyset$ or $i_{l}^{\prime} \leq i_{l}$, for all $l \in B$. On the other hand, since $\sum_{k=1}^{n} i_{k}<\sum_{k=1}^{n} i_{k}^{\prime}$, there is $s \in$ $A$ such that $i_{s}^{\prime} \geq i_{s}+1$. According to (1) and the definition of $A$ we obtain

$$
\frac{\mathcal{A}_{j}\left(i_{j}^{\prime}\right)}{\mu\left(E_{s}\right)^{i_{s}^{\prime}-1} \phi^{-1}\left(\frac{\mu\left(E_{s}\right)}{\mu(E)}\right)} \geq \frac{\mathcal{A}_{j}\left(i_{j}\right)}{\mu\left(E_{s}\right)^{i_{s}} \phi^{-1}\left(\frac{\mu\left(E_{s}\right)}{\mu(E)}\right)} \geq \frac{\mathcal{A}_{j}\left(i_{j}\right)}{M \mathcal{A}_{s}\left(i_{s}\right)} \geq M^{\prime},
$$

as $\mu(E) \rightarrow 0$, for some constant $M^{\prime}>0$. Therefore, $<i_{k}^{\prime}>$ cannot be balanced.

(b) Suppose $i_{j}^{\prime}<i_{j}$ for some $j \in B$. From (a), (1) and the definition of balanced $n$ tuple, we obtain for each $l \in A$,

$$
\frac{\mathcal{A}_{j}\left(i_{j}^{\prime}\right)}{\mu\left(E_{l}\right)^{i^{\prime}{ }_{l}-1} \phi^{-1}\left(\frac{\mu\left(E_{l}\right)}{\mu(E)}\right)} \geq \frac{\mathcal{A}_{j}\left(i_{j}-1\right)}{M \mathcal{A}_{l}\left(i_{l}\right)} \geq M^{\prime} \frac{\mu\left(E_{j}\right)^{i_{l}-1} \phi^{-1}\left(\frac{\mu\left(E_{j}\right)}{\mu(E)}\right)}{\mathcal{A}_{l}\left(i_{l}\right)} \rightarrow \infty,
$$

as $\mu(E) \rightarrow 0$. Therefore $<i_{k}^{\prime}>$ cannot be balanced. 
Given a balanced integer, the above lemma gives us a necessary condition which must satisfy the next balanced integer. The following example shows that the conditions of Lemma 4.3 are not sufficient to get a balanced $n$-tuple.

Example 4.4. Define $\varphi(x)=x^{3}(1+|\ln x|), x>0$, and $\varphi(0)=0$. Consider two points $x_{1}, x_{2}$ with $\mu\left(E_{1}\right)=\delta^{4 / 3}, \mu\left(E_{2}\right)=\delta^{1 / 3}$, and $A_{1}=A_{2}=[0,1]$. The 2-tuple $<0,1>$ is balanced. Here, the set $A(<0,1>)=\{0\}$, however $<1,1>$ is not a balanced 2-tuple. In fact, if $\left\langle i_{k}>=<0,1>\right.$ we obtain

$$
\min _{1 \leq k \leq 2}\left\{\mu\left(E_{k}\right)^{i_{k}-1}\left(\frac{\mu\left(E_{k}\right)}{\mu(E)}\right)\right\}=\min \left\{\frac{\phi^{-1}(\delta)}{\delta^{4 / 3}}, \phi^{-1}(1)\right\}+o(1) \rightarrow \phi^{-1}(1),
$$

as $\delta \rightarrow 0$. Since $\mathcal{A}_{2}\left(i_{2}\right)=o\left(\mathcal{A}_{1}\left(i_{1}\right)\right)$ and $\mathcal{A}_{1}\left(i_{1}\right)=o(1)$, as $\delta \rightarrow 0$, we have

$$
\frac{\sum_{k=1}^{2} \mathcal{A}_{k}\left(i_{k}\right)}{\min _{1 \leq k \leq 2}\left\{\mu\left(E_{k}\right)^{i_{k}-1} \phi^{-1}\left(\frac{\mu\left(E_{k}\right)}{\mu(E)}\right)\right\}}=o(1), \quad \text { as } \delta-0 .
$$

So $<0,1>$ is a balanced 2-tuple, $A(<0,1>)=\{0\}$, and $<1,1>$ is the next 2-tuple generated by the algorithm. For $\left\langle i_{k}\right\rangle=\langle 1,1>$ we have

$$
\frac{\mathcal{A}_{2}\left(i_{2}\right)}{\min _{1 \leq k \leq 2}\left\{\mu\left(E_{k}\right)^{i_{k}-1} \phi^{-1}\left(\frac{\mu\left(E_{k}\right)}{\mu(E)}\right)\right\}} \geq \frac{\mathcal{A}_{2}\left(i_{2}\right)}{\phi^{-1}\left(\frac{\mu\left(E_{1}\right)}{\mu(E)}\right)} \rightarrow \infty, \text { as } \delta \rightarrow 0 .
$$

Thus $<1,1>$ is not a balanced 2-tuple.

Next, we establish an algorithm which gives all balanced $n$-tuples. First, we observe that $\langle 0\rangle$ is a balanced $n$-tuple. In fact, since $\varphi^{-1}$ is a concave positive function on $\mathbb{R}_{+}$ with $\varphi^{-1}(0)=0$, we have $\varphi^{-1}(x) \geq \varphi^{-1}(1) x$, for $x \leq 1$. This yields

$$
\frac{\mu\left(E_{j}\right)}{\phi^{-1}\left(\frac{\mu(E)}{\mu\left(E_{k}\right)}\right) \phi^{-1}\left(\frac{\mu\left(E_{j}\right)}{\mu(E)}\right)} \leq \frac{\mu(E)}{\left(\phi^{-1}(1)\right)^{2}}, \quad 1 \leq j, k \leq n .
$$

Algorithm. Let $v_{q}$ be a balanced integer and let $<i_{k}^{(v q)}>$ be the corresponding balanced $n$-tuple. To build the next $n$-tuple, $\left\langle i_{k}^{(v q+1)}>\right.$, put $i_{k}^{(v q+1)}=i_{k}^{(v q)}+1$ for $k \in A\left(<i_{k}^{(v q)}>\right)$ and $i_{k}^{\left(v_{q}+1\right)}=i_{k}^{\left(v_{q}\right)}$ for $k \in B\left(<i_{k}^{(v q)}>\right)$.

The following lemma shows that all balanced $n$-tuples are contained in the set of $n$ tuples generated by the algorithm.

Lemma 4.5. if $<i_{k}>$ is a balanced $n$-tuple with $\sum_{k=1}^{n} i_{k}=q$, then the algorithm generates all the balanced $n$-tuple $<i_{k}^{*}>$ with $\sum_{k=1}^{n} i_{k}^{*}>q$.

Proof. Suppose $\left\langle i_{k}^{*}>\right.$ is a balanced $n$-tuple with $\sum_{k=1}^{n} i_{k}^{*}=m>q$, and the $n$-tuple $<i_{k}^{(m)}>$ is not balanced. Since $\sum_{k=1}^{n} i_{k}^{*}=\sum_{k=1}^{n} i_{k}^{(m)}$, there exist $r$ and $s$ such that $i_{r}^{(m)}>i_{r}^{*}$ and $i_{s}^{*}>i_{s}^{(m)}$. By definition of balanced integer we have

$$
\mathcal{A}_{r}\left(i_{r}^{(m)}-1\right)=O\left(\mathcal{A}_{r}\left(i_{r}^{*}\right)\right)=o\left(\mu\left(E_{s}\right)^{i_{s}^{*}-1} \phi^{-1}\left(\frac{\mu\left(E_{s}\right)}{\mu(E)}\right)\right),
$$


and (1) implies $\quad \mu\left(E_{s}\right)^{i_{s}^{*}-1} \phi^{-1}\left(\frac{\mu\left(E_{s}\right)}{\mu(E)}\right) \leq K \phi^{-1}(1) \mathcal{A}_{s}\left(i_{s}^{*}-1\right) . \quad$ So, $\mathcal{A}_{r}\left(i_{r}^{(m)}-1\right)=o\left(\mathcal{A}_{s}\left(i_{s}^{(m)}\right)\right)$.

On the other hand, since $m>q$, Lemma 4.3 implies $i_{r}^{*} \geq i_{r}$, so $i_{r}^{(m)}>i_{r}$. Therefore $\mathcal{A}_{r}\left(i_{r}^{(m)}-1\right)$ is maximal in a previous step of the algorithm, i.e., there exists $m^{\prime}, q \leq$ $m^{\prime}<m$, such that $\mathcal{A}_{r}\left(i_{r}^{(m)}-1\right)$ is maximal of $<\mathcal{A}_{k}\left(i_{k}^{\left(m^{\prime}\right)}\right)>$. Since the exponents $i_{k}^{(m)}$ are nondecreasing,

$$
\mathcal{A}_{s}\left(i_{s}^{(m)}\right)=O\left(\mathcal{A}_{s}\left(i_{s}^{\left(m^{\prime}\right)}\right)\right)=O\left(\mathcal{A}_{r}\left(i_{r}^{(m)}-1\right)\right)
$$

which contradicts (9).

Remark 4.6. If we assume the additional condition $\varphi^{-1}(x) \varphi^{-1}(1 / x) \geq c>0$ for $x>0$, given a balanced $n$-tuple $\left\langle i_{k}>\right.$, it is easy to see that the $n$-tuple $\left\langle i_{k}^{\prime}>\right.$ defined by $i_{k}^{\prime}=i_{k}+1$ for $k \in A\left(<i_{k}^{(v q)}>\right)$, and $i_{k}^{\prime}=i_{k}$ for $k \in B\left(<i_{k}^{(v q)}>\right)$, is balanced. It give us an algorithm that generates the infinite sequences of all balanced $n$-tuples.

Let $P C^{m}(X)$ be the class of functions with derivatives up to order $m-1$ and with bounded piecewise continuous $m^{\text {th }}$ derivative on $X$.

Next, we prove the following auxiliary lemma.

Lemma 4.7. Let $<i_{k}>$ be an ordered $n$-tuple of nonnegative integers. Suppose $h \in P C^{m}$ $(X)$, where $m=\max \left\{i_{k}\right\}$ and $h^{(j)}\left(x_{k}\right)=0,0 \leq j \leq i_{k}-1,1 \leq k \leq n$. Then

$$
\|h\|_{\phi, E}=O\left(\max \left\{\mathcal{A}_{k}\left(i_{k}\right)\right\}\right) .
$$

Proof. Expanding $h$ by the Taylor polynomial at $x_{k}$ up to the order $n$, we obtain

$$
h(x)=\sum_{k=1}^{n} h^{\left(i_{k}\right)}\left(\xi_{k}\right) \frac{\left(x-x_{k}\right)^{i_{k}}}{i_{k} !} \chi_{E_{k}}(x), \quad x \in E,
$$

where $\xi_{k}$ is between $x$ and $x_{k}$. The change of variable $x-x_{k}=\epsilon y, y \in A_{k}$, yields

$$
\|h\|_{\phi, E}=\inf \left\{\lambda>0: \sum_{k=1}^{n} \int_{A_{k}} \mu\left(E_{k}\right) \phi\left(\frac{\left|h^{\left(i_{k}\right)}\left(\xi_{k}\right)\right| \frac{\mu\left(E_{k}\right)^{i_{k}}\left|\gamma^{i_{k}}\right|}{i_{k} !}}{\lambda}\right) \frac{d y}{\mu(E)} \leq 1\right\} .
$$

For

$$
\lambda:=M \sum_{j=1}^{n} \frac{\mu\left(E_{j}\right)^{i_{j}}}{\phi^{-1}\left(\frac{\mu(E)}{n \mu\left(E_{j}\right)}\right)}
$$

where $M=\max _{1 \leq k \leq n}\left\{\frac{1}{i_{k} !} \max _{x \in X}\left\{\left|h^{\left(i_{k}\right)}(x)\right|\right\} \max _{y \in A_{k}}\left\{|y|^{i_{k}}\right\}\right\}$, we obtain

$$
\sum_{k=1}^{n} \int_{A_{k}} \mu\left(E_{k}\right) \phi\left(\frac{\left|h^{\left(i_{k}\right)}\left(\xi_{k}\right)\right| \frac{\mu\left(E_{k}\right)^{i_{k}}\left|\gamma^{i_{k}}\right|}{i_{k} !}}{\lambda}\right) \frac{d y}{\mu(E)} \leq 1 .
$$


Therefore $\|h\|_{\phi, E}=O\left(\sum_{k=1}^{n} \frac{\mu\left(E_{k}\right)^{i_{k}}}{\phi^{-1}\left(\frac{\mu(E)}{n \mu\left(E_{k}\right)}\right)}\right)$. Using the convexity of $\varphi$, we have $\frac{\phi^{-1}(x)}{n} \leq \phi^{-1}\left(\frac{x}{n}\right), x \geq 0$. So, $\|h\|_{\phi, E}=O\left(\max \left\{\mathcal{A}_{k}\left(i_{k}\right)\right\}\right)$.

If a polynomial $P \in \Pi^{N}, N+1=\sum_{k=1}^{n} i_{k}$, satisfies $P^{(j)}\left(x_{k}\right)=f^{(j)}\left(x_{k}\right), 1 \leq j \leq i_{k}-1,1 \leq$ $k \leq n$, we call it the Hermite interpolating polynomial of the function $f$ on $\left\{x_{1}, \ldots, x_{n}\right\}$.

Now, we are in condition to prove the main result in this Section.

Theorem 4.8. Let $<i_{k}>$ be a balanced $n$-tuple and $N+1=\sum_{k=1}^{n} i_{k}$. If $m=\max \left\{i_{k}\right\}$ and $f \in P C^{m}(X)$, then the best local approximation of ffrom $\Pi^{N}$ on $\left\{x_{1}, \ldots, x_{k}\right\}$ is the Hermite interpolating polynomial of $f$ on $\left\{x_{1}, \ldots, x_{n}\right\}$.

Proof Let $H \in \Pi^{N}$ be the Hermite interpolating polynomial and let $\left\{g_{E}\right\}$ be a net of best approximations of $f$ from $\Pi^{N}$ respect to $\|\cdot\|_{\varphi, E}$. From Lemma 4.7,

$$
\left\|g_{E}-H\right\|_{\phi, E}=O\left(\max \left\{\mathcal{A}_{k}\left(i_{k}\right)\right\}\right) .
$$

Using Theorem 3.1 and the equivalence of the norms in $\Pi^{N}$, we get

$$
\left\|g_{E}-H\right\|_{\infty} \leq \frac{K}{\min _{1 \leq k \leq n}\left\{\mu\left(E_{k}\right)^{i_{k}-1} \phi^{-1}\left(\frac{\mu\left(E_{k}\right)}{\mu(E)}\right)\right\}}\left\|g_{E}-H\right\|_{\phi, E .}
$$

So, the definition of balanced $n$-tuple implies $g_{E} \rightarrow H$, as $\mu(E) \rightarrow 0$.

\section{Acknowledgements}

This work was supported by Universidad Nacional de Rio Cuarto, Universi-dad Nacional de San Luis and CONICET.

\section{Author details}

'Department of Mathematics, UNRC, 5800, Río Cuarto, Argentina ${ }^{2}$ Department of Mathematics, UNSL, 5700, San Luis, Argentina

\section{Authors' contributions}

The three authors participated in the preparation of all work. All authors read and approved the final manuscript.

\section{Competing interests}

The authors declare that they have no competing interests.

Received: 14 July 2011 Accepted: 10 February 2012 Published: 10 February 2012

\section{References}

1. Krasnosel'skii, M, Rutickii, Ya: Convex Function and Orlicz Spaces. Noordhoff Groningen. (1961)

2. Chui, C, Diamond, H, Raphael, R: On best data approximation. Approx Theory Appl. 1, $37-56$ (1984)

3. Cuenya, H, Favier, S, Levis, F, Ridolfi, C: Weighted best local $|\cdot|$-approximation in Orlicz spaces. Jaen J Approx. 2(1):113-127 (2010)

4. Favier, S, Ridolfi, C: Weighted best local approximation in Orlicz spaces. Anal Theory Appl. 24(3):225-236 (2008). doi:10.1007/s10496-008-0225-y

5. Cuenya, H, Levis, F, Marano, M, Ridolfi, C: Best local approximation in Orlicz spaces. Numer Funct Anal Optim. 32(11):1127-1145 (2011). doi:10.1080/01630563.2011.590264

6. Borwein, P, Erdelyi, T: Polynomials and Polynomial Inequalities. Springer, New York (1995)

7. Ganzburg, MI: Polynomial inequalities on measurable sets and their applications. Constr Approx. 17, 275-306 (2001). doi:10.1007/s003650010020

8. Timan, FA: Theory of Approximation of Functions of a Real Variable. Pergamon Press, New York. (1963)

9. Cuenya, $H$, Levis, F: Pólya-type polinomial inequalities in $L^{p}$ spaces and best local approximation. Numer Funct Anal Optim. 26(7-8):813-827 (2005). doi:10.1080/01630560500431084 